This item was submitted to Loughborough's Research Repository by the author.

Items in Figshare are protected by copyright, with all rights reserved, unless otherwise indicated.

\title{
Three-way coupling simulation of a gas-liquid stirred tank using a multi- compartment population balance model
}

PLEASE CITE THE PUBLISHED VERSION

http://dx.doi.org/10.1515/cppm-2015-0076

\section{PUBLISHER}

(c) Walter de Gruyter $\mathrm{GmbH}$

VERSION

AM (Accepted Manuscript)

\section{PUBLISHER STATEMENT}

This work is made available according to the conditions of the Creative Commons Attribution-NonCommercialNoDerivatives 4.0 International (CC BY-NC-ND 4.0) licence. Full details of this licence are available at: https://creativecommons.org/licenses/by-nc-nd/4.0/

\section{LICENCE}

CC BY-NC-ND 4.0

\section{REPOSITORY RECORD}

Gimbun, Jolius, Shi Yan Liew, Zoltan Nagy, and Chris Rielly. 2016. "Three-way Coupling Simulation of a Gasliquid Stirred Tank Using a Multi-compartment Population Balance Model”. figshare. https://hdl.handle.net/2134/23100. 


\title{
Three-Way Coupling Simulation of a Gas-Liquid Stirred Tank using a Multi-Compartment Population Balance Model
}

\begin{abstract}
Modelling of gas-liquid stirred tanks is very challenging due to the presence of strong bubbleliquid interactions. Depending upon the needs and desired accuracy, the simulation may be performed by considering one-way, two-way, three-way or four-way coupling between the primary and secondary phase. Accuracy of the prediction on the two-phase flow generally increases as the details of phase interactions increase but at the expense of higher computational cost. This study deals with two-way and three-way coupling of gas-liquid flow in stirred tanks which were then compared with results via four-way coupling. Population balance model (PBM) based on quadrature method of moments (QMOM) was implemented in a multi-compartment model of an aerated stirred tank to predict local bubble size. The multi-compartment model is regarded as three-way coupling because the local turbulent dissipation rates and flow rates were obtained from a two-way computational fluid dynamics (CFD) simulation. The predicted twophase flows and local bubble size showed good agreement with experimental data.
\end{abstract}

Key words: Computational fluid dynamics, multi-compartment model, three-way coupling, population balance model, gas-liquid

\section{Introduction}

Gas-liquid stirred tanks are widely employed in fine-chemical manufacturing, pharmaceutical processes and biochemical fermentation. It is vital to have a good gas dispersion in gas-liquid stirred tank to achieve the desired production output. The estimated lost due to poor stirred tank design is over USD 600 million annually for pharmaceutical industry and over USD 1 billion annually for chemical industry (Kresta et al., 2015). Industrial stirred vessels still rely on empirical and semi-empirical correlations derived from laboratory experiments for scale-up and design. Such methods are currently only limited to similar geometrical designs and are incapable of providing detailed local flow phenomena. Hence, numerical simulation becomes an alternative solution to provide in depth understanding on the hydrodynamics of gas-liquid system in the stirred tank.

Numerous numerical efforts have been devoted to improve predictive accuracy of flow fields in gas-liquid stirred tanks but are hindered by the complexity of the turbulent two-phase system. One of the major challenges encountered when modelling gas-liquid system is poorly predicted bubble size distribution (BSD). It is understood bubble sizes are not homogeneous in aerated stirred tanks (as commonly assumed) due to breakage and coalescence events influenced by local turbulent quantities and spatial position (Barigou and Greaves, 1992; Laakkonen et al., 2005; 2007; Montante et al., 2008). Moreover, the accuracy of predicted polydisperse bubbles will affect the result of mass transfer rate as it concerns the interfacial area of contact between the gas and liquid phase. Thus, it is crucial to predict BSD correctly, especially for chemical and fermentation processes, where mass transfer of the two-phase system can potentially be the overall limiting step of the reaction. The mainstream method of predicting BSD is usually done by predicting gas-liquid turbulent flow via computational fluid dynamics (CFD) and employing population balance model (PBM) to account for breakage and coalescence events. 


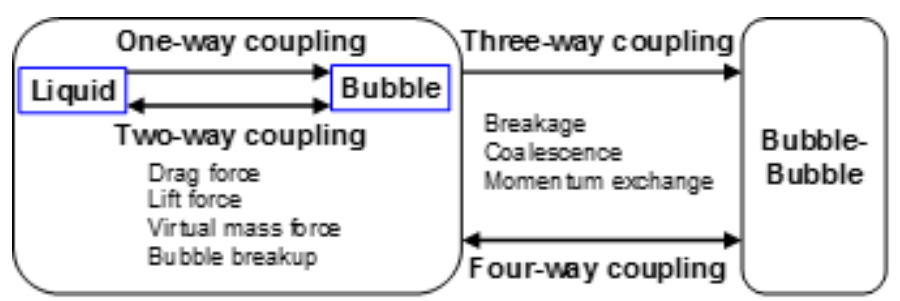

Figure 1: Illustration of phase coupling in gas-liquid modelling.

Phase coupling represents the level of interaction between gas-liquid phases usually assumed in two-phase modelling as shown in Figure 1. Earlier studies were carried out using one-way coupling; an approach that assumes only gas phase motion is affected by liquid flow. Bakker and Van den Akker (1994) and Venneker et al. (2002) have managed to obtain fair agreement with experimental result on gas hold-up but their methods were deem unrealistic as it fails to consider the effects of gas flow on liquid phase and liquid aeration height, limiting its application as a design tool. Two-way coupling on the other hand, is an approach that accounts the flow contribution from both phases on gas-liquid dispersion. It is widely applied in gas-liquid stirred tank simulation studies (e.g. Morud and Hjertager, 1996; Deen et al., 2002; Khopkar and Ranade, 2006; Sun et al., 2006; Wang et al., 2006; Scargiali et al., 2007), assuming mono-disperse bubbles throughout the tank. Mono-disperse bubbles however, indicate the absence of bubble interactions caused by coalescence and breakage events which is deem inaccurate.

Alternatively, bubble dynamic may be considered in a separate PBM using flow field information (e.g. flow rate, $\varepsilon$ and $\alpha_{g}$ ) obtained from two-way coupling simulation. This method is called threeway coupling, which employs the multi-compartment model by dividing the tank into well-mixed compartments, where turbulence dissipation rate, $\varepsilon$ and gas hold-up, $\alpha_{g}$ will be taken as a volume average value in each compartment respectively. It was reported that, the local bubble size was fairly predicted with the utilisation of PBM via method of classes (Laakkonen et al., 2006a; 2006b; 2007). However, the drawback of this method is the lack of consideration on the effect of local bubble size on gas-liquid flow field. Meanwhile, four-way coupling considers all two-way coupling, bubble dynamics and the effect of local bubble size on two-phase flow field. Fully coupled CFD-PBM solution via various derivative of the quadrature methods of moment have surfaced with satisfactory results on bubble size prediction in recent years (Gimbun et al., 2009; Buffo et al., 2012; Petitti et al., 2013). This method is promising for its high accuracy due to the implementation of PBM within CFD using user defined subroutines, but can be complicated to execute (convergence issue) and computationally expensive. A four-way coupling solution may take between several days to few weeks to simulate an aerated stirred tank, whereas the three-way coupling solution can be performed within a few minutes.

A simpler approach in modelling gas-liquid system should be seek to provide important interpretations on the two-phase as sufficiently needed without sacrificing computational cost and time. Hence, this work focuses on three-way coupling method using multi-compartment model where local conditions (i.e. local bubble size) in the tank are of interest at lower computational expense. Previous three-way coupling simulations (e.g. Alopaeus et al., 1999; Zahradnik et al., 2001; Hristov et al., 2001; Alves et al., 2002) mostly obtain their flow field data from experimental measurements or simple correlations rather than inter-compartment flow field results through two-way coupled CFD simulations. Such approaches are not considered as proper three-way coupling simulation as experimental flow field would have accounted the effects from local bubble size and limit the flexibility over other stirred tank designs. In addition, there has yet to be any three-way coupling stirred tank simulations performed using quadrature moment of methods (QMOM) and this is one of the objectives of this study. 
This paper concerns the development and validation of a multi-compartment model for the simulation of a gas-liquid stirred tank. The exchange of inter-compartment flows, the local gas hold-up and the local energy dissipation rates were estimated using flow field CFD calculations conducted assuming constant initial bubble size. PBM based on QMOM was implemented to accommodate the bubble break-up and coalescence phenomenon to predict local bubble size for each compartment. In order to validate the QMOM system, a single compartment model was initially carried out involving bubble break-up and coalescence. A sensitivity study concerning the number of quadrature approximation points used for the QMOM and their effect on the prediction accuracy were evaluated. The single compartment model is then extended into a multicompartment model for the simulation of gas-liquid aerated stirred tank (refer to supplementary data). Results from the multi-compartment model are then compared with experimental measurements Laakkonen et al. (2007) and CFD-PBM results by Gimbun et al. (2009).

\section{CFD approach for gas-liquid stirred tanks 2.1 CFD modelling of two-phase flow}

Eulerian-Eulerian multiphase model was employed in this work, whereby the continuous and disperse phases are considered as interpenetrating media, identified by their local volume fractions. The liquid volume fraction sums to unity and is governed by the following continuity equation:

$$
\frac{\partial}{\partial t}\left(\alpha_{l} \rho_{l}\right)+\nabla \cdot\left(\alpha_{l} \rho_{l} \vec{u}_{l}\right)=0
$$

where $\alpha_{l}$ is the liquid volume fraction, $\rho_{l}$ is the density, and $\vec{u}_{l}$ is the velocity of the liquid phase. The mass transfer between phases is negligibly small and hence is not included in the RHS of Eq. 1. A similar equation is solved for the volume fraction of the gas phase by replacing the subscript $l$ with $g$. The momentum balance for the liquid phase is:

$$
\frac{\partial}{\partial t}\left(\alpha_{l} \rho_{l} \vec{u}_{l}\right)+\nabla \cdot\left(\alpha_{l} \rho_{l} \vec{u}_{l} \vec{u}_{l}\right)=-\alpha_{l} \nabla P+\nabla \cdot \overline{\bar{\tau}}_{l}+\vec{F}_{l g}+\alpha_{l} \rho_{l} \vec{g}+\vec{F}_{l i f t, l}+\vec{F}_{v m, l}
$$

where $\overline{\bar{\tau}}_{l}$ is the liquid phase stress-strain tensor, $\vec{F}_{l i f t, l}$ is a lift force, $\vec{g}$ is the acceleration due to gravity, $\vec{F}_{v m, l}$ is the virtual mass force and a similar equation is solved for the gas phase as well. $\vec{F}_{\text {lg }}$ on the other hand, accounts the interaction force per unit volume of mixture between phases, mainly due to drag. An assessment conducted by Scargiali et al. (2007) concluded that the effect of virtual mass and lift force in stirred tanks are relatively negligible in comparison to drag. By adding the effects of virtual mass and lift force in their study, there was minimal increase of overall gas hold-up by $0.24 \%$ and $0.31 \%$ respectively, aside from a significant increase of unnecessary computational expense and convergence difficulties. Previous studies have also resorted to similar practice (e.g. Bakker and Van Den Akker, 1994; Morud and Hjertager, 1996; Lane et al., 2002; Kerdouss et al., 2006) and thus in this work they were omitted as well. $\vec{F}_{\mathrm{lg}}$ is represented by a simple interaction term for drag force given by:

$$
\vec{F}_{\mathrm{lg}}=-\frac{3 \alpha_{g} \alpha_{l} \rho_{l} C_{D}\left|\vec{u}_{g}-\vec{u}_{l}\right|\left(\vec{u}_{g}-\vec{u}_{l}\right)}{4 d_{b}}
$$


where $C_{D}$ is the drag coefficient and $d_{b}$ is the Sauter mean bubble diameter. Drag models tend to have a significant effect on aerated flow fields, as it relates directly to the bubble terminal rise velocity. Schiller and Naumann (1935) standard FLUENT drag model is only best suited for spherical bubbles which are by nature small in size (i.e. air-water for bubble with diameter lesser than $3 \mathrm{~mm}$ ). Thus, a modified drag model that considers non-spherical bubbles, especially those with diameter more than $3 \mathrm{~mm}$ is appropriate for realistic interpretations of the flow field. The drag model by Ishii and Zuber (1979) was selected in this work, as it takes into account the drag of distorted bubbles:

$$
C_{D}=\max \left(\frac{24}{\operatorname{Re}_{b}}\left(1+0.15 \operatorname{Re}_{b}^{0.687}\right), \min \left(\frac{2}{3} \sqrt{E_{O}}, 8 / 3\right)\right)
$$

where the $R e_{b}=\rho u_{\text {slip }} d_{b} / \mu$ and $E_{O}=g \Delta \rho d_{b}^{2} / \sigma$ are the bubble Reynolds number and Eotvos number, respectively. The slip velocity, $u_{\text {slip }}$, is given by:

$$
u_{\text {slip }}=\left|\vec{u}_{g}-\vec{u}_{l}\right|
$$

The drag for ellipsoidal bubble regime is dependent on the bubble shape through the Eotvos number, meanwhile for spherical cap regime the drag coefficient is $8 / 3$. The cap regime is negligible when the aeration rate is low in gas-liquid stirred tanks but should be accounted for large bubbles. This setting is not a standard option in FLUENT and hence was implemented using user-defined subroutine to activate the cap regime equation when bubble size becomes larger than $10.9 \mathrm{~mm}$. The effect of the local bubble volume fraction on the drag coefficient was estimated using Behzadi et al. (2004) correlation as follows:

$$
C_{D, \text { dense }}=C_{D}\left(e^{3.64 \alpha}+\alpha^{0.864}\right)
$$

where $C_{D}$ is the drag coefficient for an isolated bubble estimated using Eq. 4 , whereas $C_{D \text {,dense }}$ is for the dense dispersion of bubbles.

It is also crucial that the formation of bubble cavity behind the impeller blade is considered as the cavity behind the blade behaves in a manner similar to an isolated bubble rather than a dense bubble at high void fractions. Modelling the gas cavity can be done using the Eulerian-Eulerian multiphase model by modifying the interphase exchange; the drag coefficient is set to account the case as isolated bubbles when the void fraction is greater than 0.7 (Lane et al., 2005). An attempt to implement the dense drag model in cavity regions has resulted to the disappearances of the bubble cavity behind the blade and an over-prediction of the relative gassed power number by more than 20\% from 0.45 of Smith (2006) correlation to 0.55. Significant increase in radial velocity was also observed. However, this issue has been successfully overcome by disabling the dense drag model around the cavity by setting the model to calculate the drag for isolated bubble when the local volume fraction exceeded 0.7 .

The effects of turbulent dispersion on bubble drag coefficient may affect the gas hold-up in stirred tanks. However, Scargiali et al. (2007) found a minimal difference on gas hold-up in stirred tank with turbulent dispersion of $4.36 \%$, without turbulent dispersion of $4.35 \%$ compared to the measurement by Bombac et al. (1997) of 4.20\%. They concluded that, turbulent dispersion 
of the gas phase seems to play a negligible role in gas distribution. Hence, the effect of turbulent dispersion on drag model is omitted in this work.

\subsection{Modelling of turbulence}

This work implements two-phase realizable $k-\varepsilon$ turbulence model for gas-liquid stirred tank simulation, in which both the $k$ and $\varepsilon$ are allowed to have different values for each phase. The transport equations for the model were described in the Fluent (2005) and the standard values of the model parameters have been applied. The realizable $k-\varepsilon$ model is designated to be superior to standard $k-\varepsilon$ model at predicting turbulent quantities for flow features inhibiting strong streamline curvature, vortices and rotation (Gimbun, 2009). This is due to the introduction of a new turbulent viscosity formulation and new transport equation for dissipation rate that incorporates different model constants within the realizable $k-\varepsilon$ turbulence model.

\section{Modelling of bubble breakage and coalescence}

PBM considers the birth and death of bubbles due to breakage and coalescence events. To eliminate closure problem since integration cannot be written in terms of moment, transformed QMOM equation for the $k^{\text {th }}$ moment of a single well-mixed system is given by:

$$
\begin{aligned}
& \frac{\mathrm{d} \mu_{k}}{\mathrm{~d} t}= \underbrace{\sum_{i=1}^{N} w_{i} a\left(L_{i}\right) b\left(k, L_{i}\right)}_{\text {birth due to breakage }}+\underbrace{\frac{1}{2} \sum_{i=1}^{N} w_{i} \sum_{j=1}^{N} w_{j}\left(L_{i}^{3}+L_{j}^{3}\right)^{k / 3} \beta\left(L_{i}, L_{j}\right)}_{\text {birth due to coalescence }} \\
&-\underbrace{\sum_{i=1}^{N} w_{i} a\left(L_{i}\right) L_{i}^{k}}_{\text {death due to breakage }} \\
&-\underbrace{\sum_{i=1}^{N} w_{i} L_{i}^{k} \sum_{j=1}^{N} w_{j} \beta\left(L_{i}, L_{j}\right)}_{\text {death due to coalescence }}
\end{aligned}
$$

where $\beta\left(L_{i}, L_{j}\right), a\left(L_{i}\right)$ and $b\left(k, L_{i}\right)$ are the coalescence kernel, breakage kernel and daughter bubble distribution function, respectively. The weights $(w)$ and abscissas $(L)$ from the moments were solved using product difference algorithm (PD) by Gordon (1968). Prince and Blanch (1990) breakage and coalescence kernel was employed in this work following the validation performed earlier by Gimbun et al. (2009).

\subsection{Coalescence kernel}

The bubble coalescence kernel, $\beta\left(L_{i}, L_{j}\right)$, is given as a product of the collision frequency $\omega\left(L_{i}, L_{j}\right)$ and the bubble collision efficiency $\Lambda\left(L_{i}, L_{j}\right)$ (Prince and Blanch, 1990):

$\beta\left(L_{i}, L_{j}\right)=\omega\left(L_{i}, L_{j}\right) \Lambda\left(L_{i}, L_{j}\right)$

Bubble coalescence in turbulent regime may occur due to collisions driven by turbulent and buoyancy. In turbulent flow, bubble collision can occur due to random bubble movement and large velocity gradient in the mean flow. Whereas, buoyancy driven collision can occur due varying bubble sizes having different rise velocities. Thus, the bubble collision frequency for a Newtonian fluid can be modelled following the approach proposed by Prince and Blanch (1990): 


$$
\omega\left(L_{i}, L_{j}\right)=\underbrace{\pi\left(\frac{L_{i}}{2}+\frac{L_{j}}{2}\right)^{2}\left[u_{t}^{2}\left(L_{i}\right)+u_{t}^{2}\left(L_{j}\right)\right]^{0.5}}_{\text {turbulent }}+\underbrace{\pi\left(\frac{L_{i}}{2}+\frac{L_{j}}{2}\right)^{2}\left|u_{\infty}\left(L_{i}\right)-u_{\infty}\left(L_{j}\right)\right|}_{\text {bouyancy }}
$$

where $u_{t}\left(L_{i}\right)$ is the turbulent velocity in the inertial range of isotropic turbulence (Rotta, 1972):

$u_{t}\left(L_{i}\right)=1.4 \varepsilon^{1 / 3} L_{i}^{1 / 3}$

and $u_{\infty}\left(L_{i}\right)$ is the rise velocity of bubble given as a function of bubble size (e.g. using the method by Clift et al. (1978)):

$u_{\infty}\left(L_{i}\right)=\left(2.14 \frac{\sigma}{\rho_{l} L_{i}}+0.505 g L_{i}\right)^{0.5}$

Bubble collision efficiency, $\Lambda\left(L_{i}, L_{j}\right)$ is the probability of coalescence likely to occur during a bubble-bubble collision between sizes $L_{i}$ and $L_{j}$. Prince and Blanch (1990) describes the occurrence of coalescence between two bubbles in turbulent flows in three steps, which are collision, film draining and film rupture. Bubbles that successfully collide will entrap a thin film of liquid in between bubble boundaries. For a sufficient period of time, the liquid film will drain until a critical thickness is reached before rupturing, resulting to coalescence. Thus the bubble collision efficiency is given as a function of film drainage and bubble-bubble contact times as described in the following (Prince and Blanch, 1990):

$\Lambda\left(L_{i}, L_{j}\right)=\exp \left(\frac{-\ln \left(h_{o} / h_{f}\right) \sqrt{\left(L_{i j} / 2\right)^{3} \rho_{l} / 16 \sigma}}{\left(L_{i j} / 2\right)^{2 / 3} / \varepsilon^{1 / 3}}\right)$

where $L_{i j}=2\left(1 / L_{i}+1 / L_{j}\right)^{-1}, h_{o}$ is the initial film thickness and $h_{f}$ is the final thickness at which the film rupture occurs. A value of $10^{-4} \mathrm{~m}$ for $h_{o}$ and a value of $10^{-8} \mathrm{~m}$ for $h_{f}$ from Prince and Blanch (1990) was used throughout this work.

\subsection{Breakage kernel}

Prince and Blanch (1990) breakage kernel considers eddies with size larger than 0.2 times bubble diameter and eddy velocities larger than critical velocity, $u_{c i}$ to be significantly affecting overall break-up rate. The breakage kernel $a\left(L_{i}\right)$, is given as a product of the collision rate of bubbles with turbulent eddies, $\theta_{i e}$, and the break-up efficiency, $\kappa_{i}$ (Prince and Blanch, 1990):

$a\left(L_{i}\right)=\theta_{i e} \kappa_{i}$

The collision rate of bubbles with turbulent eddies is given by (Kennard, 1938): 


$$
\theta_{i e}=n_{i} n_{e} S_{i e}\left(u_{t}\left(L_{i}\right)^{2}+u_{t}\left(L_{e}\right)^{2}\right)^{0.5}
$$

where $n_{i}, n_{e}$ and $S_{i e}$ are the number of bubbles per unit volume, number of eddies per unit volume and collision cross-sectional area respectively. $u_{t}\left(L_{i}\right)$ is the turbulent velocity in the inertial range of isotropic turbulence given by Eq. 10 and the eddy velocity, $u_{t}\left(L_{e}\right)$ of eddy size, $L_{e}$ is also calculated analogously using the same Eq. 10. The eddy size is expressed using Kolmogorov (1941) theory of isotropic turbulence as $L_{e}=\left(v_{l}^{3} / \varepsilon\right)^{1 / 4}$.

$$
\kappa_{i}=\exp \left(u_{c i}^{2} / u_{t}\left(L_{e}\right)^{2}\right)
$$

where the $u_{c i}$ is the critical eddy velocity necessary to break a bubble of diameter $L_{i}$, given by Shimizu et al. (2000):

$$
u_{c i}=\left(\frac{\sigma}{L_{i} \rho_{l}}\right)^{0.5}
$$

In QMOM, the daughter BSD function, $b\left(k, L_{i}\right)$ determines the corresponding moments of daughter particles, $L_{i}$ formed from any breakage event. A uniform breakage function was

$$
b\left(k, L_{i}\right)=L_{i}^{k} \frac{6}{k+3}
$$

Although, the possibility of non-binary breakage for liquid-liquid systems may occur where internal viscosity of the dispersed phase can produce multiple daughter drops, the assumption of binary breakage is still valid as air viscosity is low and thus the bubbles cannot form an extremely elongated shape (Andersson and Andersson, 2006). In addition, Andersson and Andersson (2006) study has reported that $95 \%$ of bubble break-ups occur due to binary breakage.

\section{Results and discussion}

\subsection{Validation of CFD simulation}

Gas-liquid CFD simulation was conducted to obtain flow field results (i.e. local turbulence dissipation rate and local gas hold-up) and inter-compartment fluxes across the stirred tank for multi-compartment modelling. A study by Laakkonen et al. (2007) on a $14 \mathrm{~L}$ aerated stirred tank agitated using a Rushton turbine was adopted for both the validation and multi-compartment modelling. The stirred tank is considered to be operating in a fully dispersed regime ( $\left.N>N_{\mathrm{CD}}\right)$ for better gas dispersion. Gas was injected through a sparger ring at a flow rate of 0.7 VVM which is treated as a continuous source of gas (velocity inlet) in the CFD simulation. The CFD simulation was performed by assuming a uniform bubble diameter of $d_{32}=2 \mathrm{~mm}$ throughout the tank based on Laakkonen et al. (2007) work. The bubble size of $2 \mathrm{~mm}$ was chosen based on Laakkonen et al. 
(2007) measurement of bubble size around the impeller region, which represents about $37 \%$ of the gas hold-up and over $90 \%$ of the turbulent dissipation rate in the whole tank. The stirred tank grid was prepared with a headspace to accommodate the liquid expansion due to aeration. The liquid surface was set as a freely expandable liquid surface and the top of the headspace region was set as a pressure outlet as illustrated in Figure 3 while the tank bottom is a no-slip wall. The mass balance between the gas outflow at the outlet boundary (above the headspace region) and the gas inflow at the sparger were conserved within $0.1 \%$ in agreement. The impeller movement was modelled using multiple reference frame technique. A time averaging of RANS-Favre averaged two-phase model was applied in this study. Transient solvers with second-order spatial interpolation scheme were also employed to minimize the amount of numerical diffusion. The iteration residual was set to fall below $1 \times 10^{-4}$ at each time step to achieve good convergence. A time step of $0.005 \mathrm{~s}$ was employed. The volume average of the gas void fraction in the rotating zone (impeller region) was used to indicate when the steady-state has been obtained; the iterations in the transient simulation were only halted once a constant value was obtained.

The CFD setup was set at impeller speed, $N=513$ RPM and gas flowrate, $Q=0.7$ VVM to enable comparison with Deen (2001) flow field measurements using Laakkonen et al. (2007) tank dimensions. It is noted that Deen (2001) experiment was performed at $F_{l g}=0.029$, whereas Laakkonen et al. (2007) work was performed at $F_{l g}=0.022$. Although, the comparison of the mean and turbulence flows can be normalised using tip velocity, $V_{\text {tip }}$ to ensure these quantities were independent of the impeller speed (i.e. allowing comparisons across different $N$ ), in the case of gas-liquid stirred tank it is necessary to ensure that aeration number remains the same for fair comparison. Prior to final grid selection, a grid dependent study was performed using three different grid densities denoted as coarse (165k cells), intermediate (225k cells) and fine (335k cells), similar to the one by Gimbun et al. (2009) (refer supplementary data Figure S2). The main differences between the three grids were the number of nodes placed along the impeller blade height; 6 nodes for the coarse grid, 11 nodes for the intermediate grid, 13 nodes for the fine grid. It was found that the domain consisting 225k cells yielded a grid independent solution (refer supplementary data Figure S3), and hence it was used for the remainder of this work.

The CFD results obtained were time-averaged over all blade angles and normalised using $V_{\text {tip }}$ for easier comparison. Prediction on the radial and axial velocities for both gas and liquid phase are shown in Figure 4 whereby the CFD simulation showed reasonable agreement with PIV measurement. The predicted turbulent kinetic energy was also in agreement with Deen (2001)'s experimental data as shown in Figure S4A and Figure S4B. Discrepancy between the measured and predicted value is due to the fact that Deen (2001) only provide 2D PIV measurement, and the kinetic energy is estimated according to Zhou and Kresta (1996) pseudo-isotropic assumption whereby $k=4 / 3\left(u^{\prime 2}+v^{\prime 2}\right)$. The pseudo-isotropic assumption can be used to estimate the turbulent kinetic energy in the case when only two velocities components are available. Although the correlation assumed a pseudo-isotropic turbulence flow, they do not imply the turbulent to be isotropic because the $u^{\prime}$ and $v^{\prime}$ can vary quite significantly (Khan et al., 2006).

The two-phase CFD simulation was successfully validated, hence a CFD simulation flow field with respect to Laakkonen et al. (2007) operating condition ( $N=700$ RPM and $Q=0.7$ VVM) was performed for the multi-compartment model. Predictions of the gas hold-up and turbulent dissipation rate from the CFD simulation were shown in 5 and 6. A fully dispersed flow pattern can be observed with the gas circulating around the lower circulation loop. There is an observable region of lower gas hold-up near the tank bottom. Experimental observations often made through the tank wall, indicate in this region of bubbles tend to be fairly well dispersed, circulating near the tank bottom. This feature was expected considering $N>N_{\mathrm{CD}}$ and has been reproduced fairly 
successfully by the CFD simulation. Flow field information obtained from the CFD simulation was used to develop the multi-compartment model in this work.
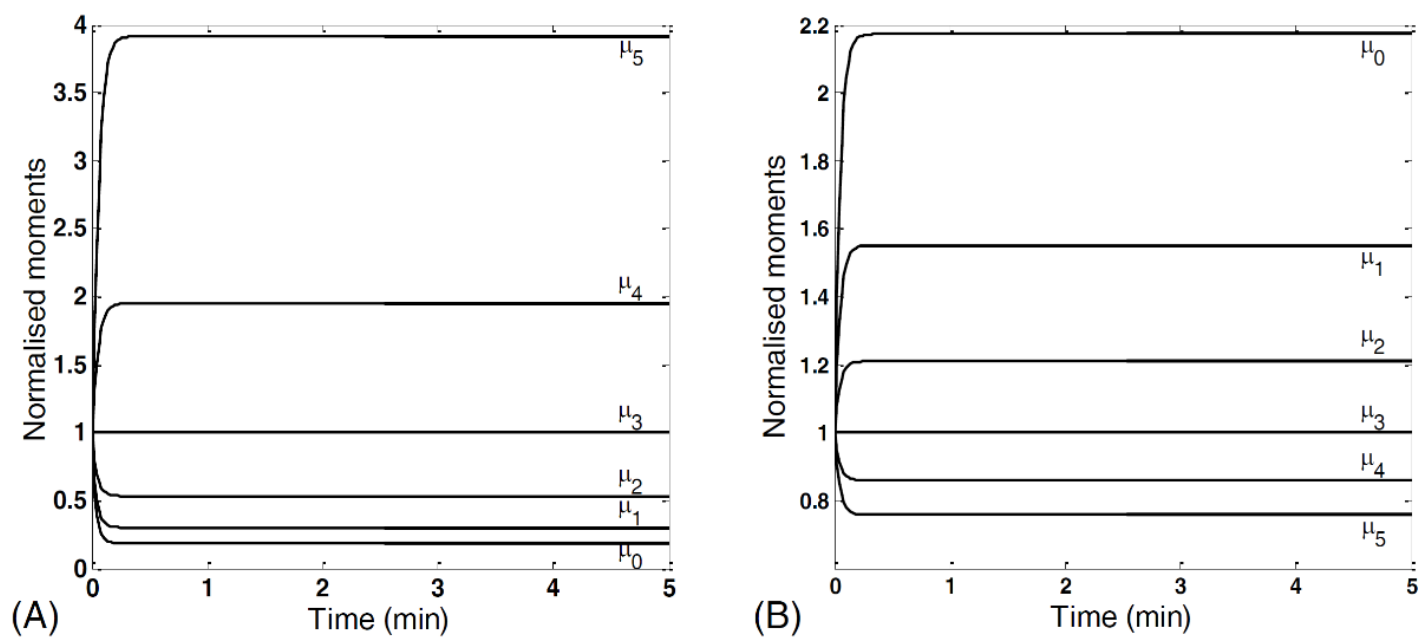

Figure 2: Evolution of moments for bubble coalescence and breakage problem, (A) Coalescence Breakage dominated case $2, \varepsilon=1.18 \mathrm{~m}^{2} / \mathrm{s}^{3}$, lognormal distribution parameter $\left(d_{\text {mean }}\right.$ initial $=5$ $\mathrm{mm})$.

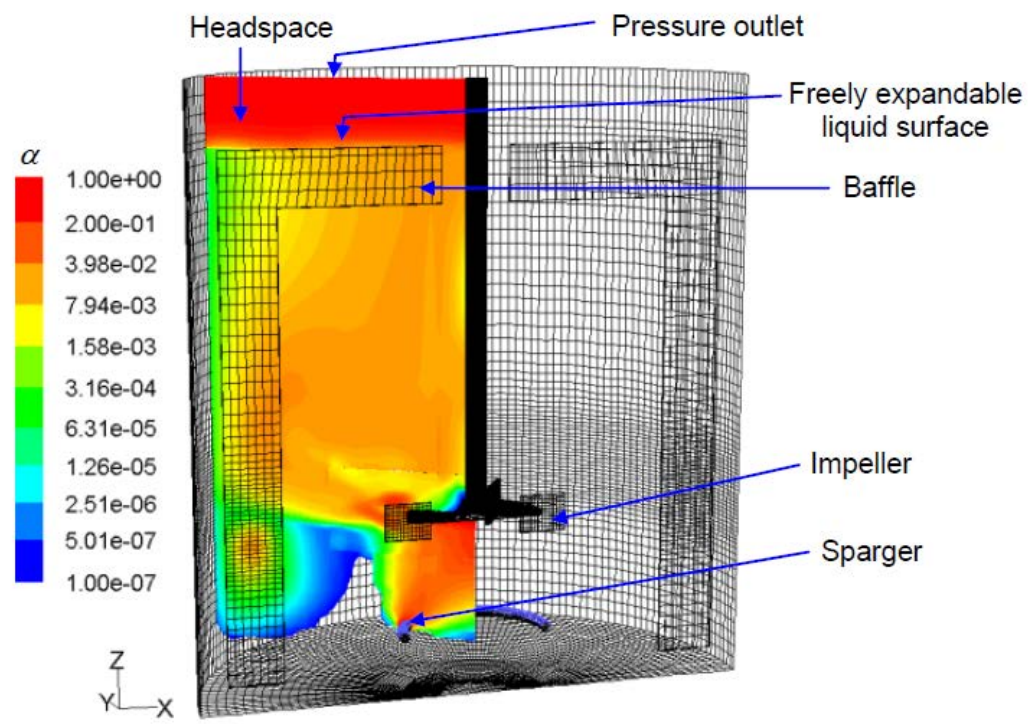

Figure 3: Boundary condition of gas-liquid stirred tank simulation. Also shown is the 340 instantaneous contour of gas hold-up. 

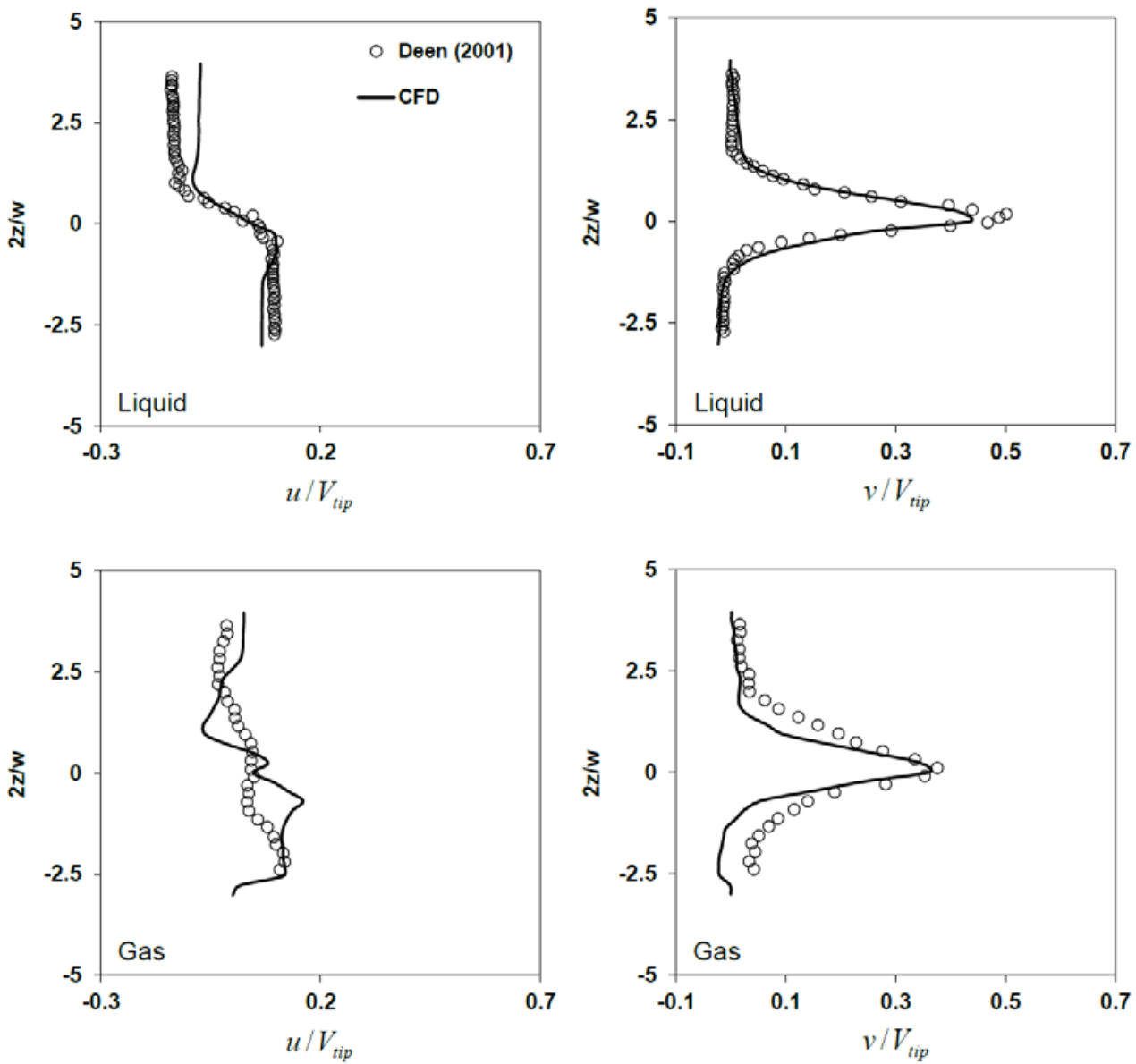

Figure 4: Prediction of liquid and gas phase axial $(u)$ and radial velocity $(v)$ at $r / R=0.37$. Experimental data is adopted from Deen (2001).

\subsection{Multi-compartment model and comparison with CFD-PBM and experiment}

Prior to the multi-compartment model, a single compartment simulation was carried out to test the reliability of the PD-QMOM response on the bubble dynamics to changes in turbulence dissipation rate, gas flow rate and initial bubble size. The simulation of the single compartment (refer to supplementary data) is then extended to the multi-compartment simulation of aerated stirred tank. Flow field data obtained from CFD results was used to divide the tank into a number of homogeneous and well-mixed compartments. The connectivity between each compartment is also determined by the flow direction obtained from two-way coupling CFD simulation. In order to divide the tank, a new mesh of the vessel consisting 12 compartments was prepared based on the CFD predicted flow patterns. The flow fields obtained from the gas-liquid CFD simulation was interpolated into the new mesh for easier data interpretation as shown in Figure 5. The compartments were split by taking into account the three major regions in a stirred tank, i.e. upper recirculation loop, impeller discharge region and lower recirculation loop.

The compartments were prepared in a way that the flow was only allowed to move in one direction at each interface responsible for separating the compartments. Figure 6A shows the vector map of the gas flow extracted from the CFD simulation which is taken as a basis to construct the compartments as shown in Figure 6B. The criteria of one direction flow for each compartment interface were all satisfied except for compartments 2 and 3 thus, manual adjustments were made on both the compartments to satisfy the inter-compartment mass balance. 
The liquid turbulence dissipation rates and the inter-compartment gas flow rates were obtained from averaging the detailed CFD results azimuthally over compartment volumes or areas, respectively. The gas flow between the compartments are obtained by reporting the fluxes (mass flow rate) through each interface in the CFD simulation. The exchanging gas flow rates of each compartment do not exactly balance (with difference up to $10^{-6} \mathrm{~kg} / \mathrm{s}$ ), possibly due to interpolation error and the fact that the CFD result was obtained from a transient simulation. Therefore, the inter-compartment flows were adjusted (balanced) manually in order to make the multi-compartment model satisfy the gas mass balance. A multi-compartment simulation with imbalanced inter-compartment flow rates would result in a different distribution of third moments (related to gas hold-up described in Eq. S6) to those obtained from the CFD simulation. It was made clear from the supplementary data on a validation performed using single compartment simulation that the third moment should be strictly preserved, unless there is a change in the local gas-hold up.

The number density of bubbles in each compartment was determined by the volume averaged gas hold-up. The initial BSD for each compartment were assumed to follow the lognormal distribution with a geometric mean diameter of $2 \mathrm{~mm}$ and standard deviation of 0.2 . The calculation for the bubble number density in each compartment were performed following the method described in the example as shown in Table S3, using the information of gas hold-up from the CFD simulation. The turbulence dissipation rates and inter-compartment gas flow rates were shown in Table 1.

Table 1: Parameter for the multi-compartment PBM.

\begin{tabular}{|c|c|c|c|c|c|c|c|}
\hline Compartment & $\begin{array}{c}\text { Volume } \\
\left(\mathrm{cm}^{3}\right)\end{array}$ & $\begin{array}{c}\alpha_{g} \\
\times 100\end{array}$ & $\begin{array}{c}\varepsilon \\
\left(\mathrm{m}^{2} / \mathrm{s}^{3}\right)\end{array}$ & $\begin{array}{l}\text { Gas flow } \\
\text { direction }\end{array}$ & $\begin{array}{l}\text { Gas flow } \\
\text { rate }\left(\mathrm{cm}^{3} / \mathrm{s}\right)\end{array}$ & $\begin{array}{l}\text { Gas flow } \\
\text { direction }\end{array}$ & $\begin{array}{c}\text { Gas flow } \\
\text { rate }\left(\mathrm{cm}^{3} / \mathrm{s}\right)\end{array}$ \\
\hline 1 & 635.85 & 7.56 & 11.35 & $q_{11 \text { to } 12}$ & 28.08 & $q_{6 \mathrm{to7}}$ & 0.00 \\
\hline 2 & 1502.22 & 1.16 & 2.57 & $q_{9 \mathrm{to} 12}$ & 57.78 & $q_{2 \mathrm{to} 8}$ & 108.48 \\
\hline 3 & 552.35 & 1.56 & 0.40 & $q_{10 \mathrm{to} 11}$ & 25.47 & $q_{2 \text { to } 9}$ & 46.36 \\
\hline 4 & 394.21 & 0.07 & 0.05 & $q_{8 \mathrm{to} 10}$ & 41.23 & $q_{8 \mathrm{to} 9}$ & 67.25 \\
\hline 5 & 863.14 & 0.02 & 0.12 & $q_{9 t 011}$ & 55.83 & $q_{1 \mathrm{to} 12}$ & 6.21 \\
\hline 6 & 2425.29 & 0.00 & 0.05 & $q_{2 \mathrm{to} 3}$ & 3.33 & $q_{1 \mathrm{to} 2}$ & 154.84 \\
\hline 7 & 1043.64 & 1.53 & 0.06 & $q_{4 \text { to2 }}$ & 3.33 & $q_{7 \mathrm{to} 1}$ & 161.05 \\
\hline 8 & 1370.65 & 1.44 & 0.29 & $q_{3 \mathrm{to} 4}$ & 2.92 & $q_{\text {out12 }}$ & 92.07 \\
\hline 9 & 977.86 & 3.92 & 0.05 & $q_{3 \mathrm{to} 5}$ & 0.41 & $q_{\text {out11 }}$ & 53.22 \\
\hline 10 & 1938.08 & 0.47 & 0.06 & $q_{4 \text { to } 7}$ & 0.00 & $q_{\text {out } 10}$ & 15.76 \\
\hline 11 & 1378.62 & 2.14 & 0.06 & $q_{6 \mathrm{to} 4}$ & 0.41 & $q_{s p r}$ & 161.05 \\
\hline 12 & 2427.41 & 3.72 & 0.03 & $q_{5 \text { to6 }}$ & 0.41 & & \\
\hline
\end{tabular}

The sparger is modelled as a constant source of bubbles (a nucleation term) with uniform diameter of $5.5 \mathrm{~mm}$, following the experimental measurements by Laakkonen et al. (2007). The gas flow rate of $1.6 \times 10^{-4} \mathrm{~m}^{3} / \mathrm{s}(0.7 \mathrm{VVM})$ was set to match Laakkonen et al. (2007)'s experiment. Table 2 shows the rate of the moments introduction at the sparger (i.e. compartment no. 7) which is calculated using the following equation:

$$
S_{\text {sparger }, k}=\frac{6 q_{s p r} L^{k-3}}{\pi \overline{\mathrm{V}_{7}}}
$$

Table 2: Rate of moments introduction at sparger.

\begin{tabular}{ll}
\hline$S_{\text {sparger, o }}\left(/ \mathrm{m}^{3} \mathrm{~s}\right)$ & 1771417.33 \\
$S_{\text {sparger, }, 1}\left(\mathrm{~m} / \mathrm{m}^{3} \mathrm{~s}\right)$ & 9742.80 \\
\hline
\end{tabular}




\begin{tabular}{ll}
\hline$S_{\text {sparger, 2 }}\left(\mathrm{m}^{2} / \mathrm{m}^{3} \mathrm{~s}\right)$ & 53.59 \\
$S_{\text {sparger, 3 }}\left(\mathrm{m}^{3} / \mathrm{m}^{3} \mathrm{~s}\right)$ & 0.29 \\
\hline
\end{tabular}

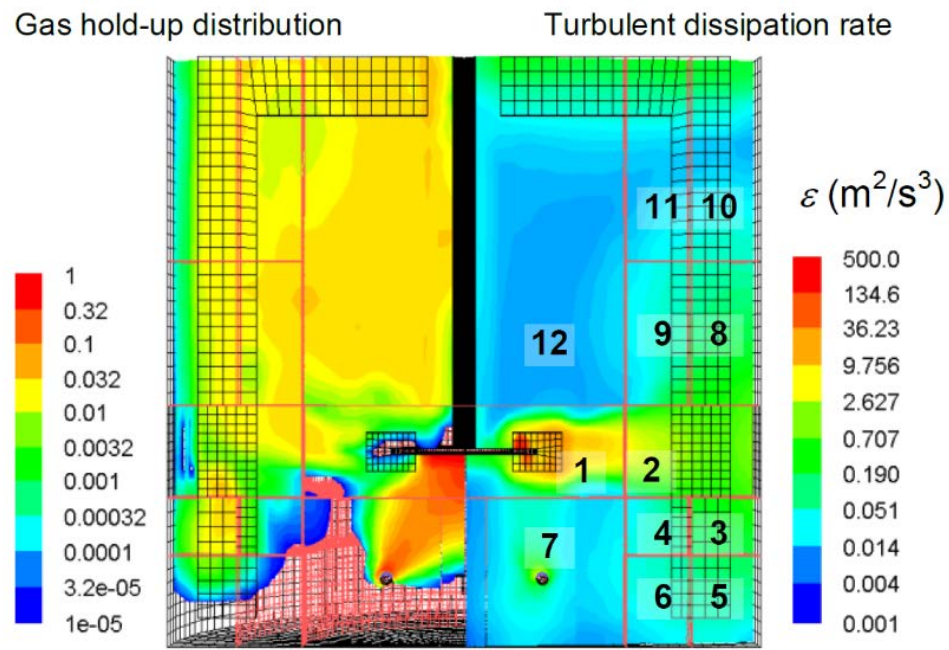

Figure 5: Contour of $\alpha_{g}$ and liquid $\varepsilon\left(\mathrm{m}^{2} / \mathrm{s}^{3}\right)$ after interpolation to the 12 block mesh, the number at each block represent the compartment number.

(A)

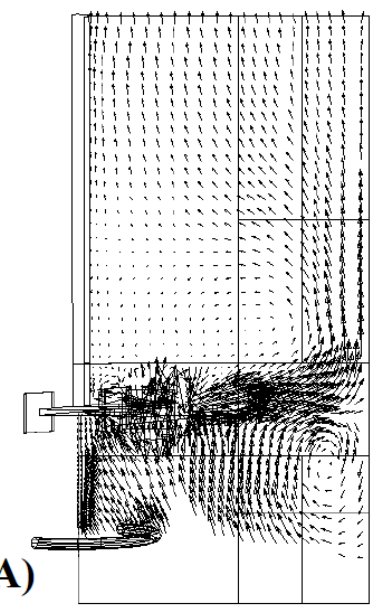

(B)

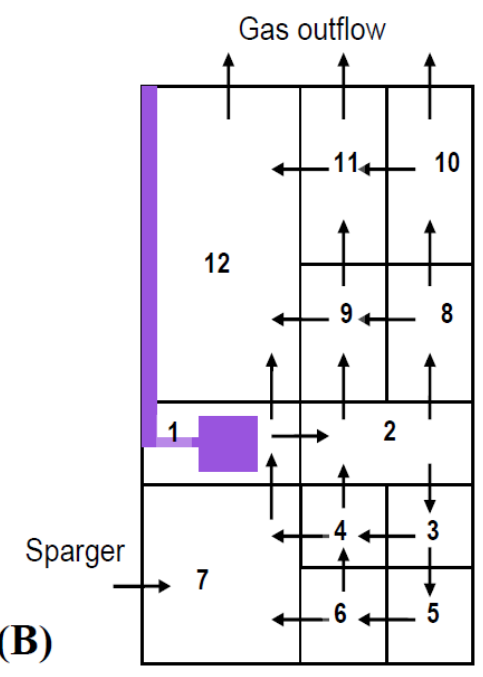

Figure 6: (A) Gas velocity vector in between two baffles obtained from CFD simulation, (B) Compartment connectivity for half of the tank based on inter-compartmental gas flow rate. Symmetry around the impeller axis is assumed.

The gas flow leaving at the top of the tank is also taken into consideration in the multicompartment model. The CFD simulation result of the gas flow rate leaving the top liquid surface (i.e. which is $161.1 \mathrm{~cm}^{3} / \mathrm{s}$ ) was also adjusted to match the gas inflow at the sparger. The multi-compartment model is developed based on the compartment flow connectivity from the CFD simulation as shown in Table 1 and Figure 6. The multi-compartment moment equations that were used are as follows:

$\frac{\partial \mu_{1, k}}{\partial t}=\frac{1}{V_{1}}\left[\frac{q_{7 \mathrm{to} 1} \mu_{7, k}}{\alpha_{7}}-\left(q_{1 \mathrm{to} 2}+q_{1 \mathrm{to} 12}\right) \frac{\mu_{1, k}}{\alpha_{1}}\right]+B-D$ 


$$
\begin{aligned}
& \frac{\partial \mu_{2, \mathrm{k}}}{\partial t}=\frac{1}{\overline{V_{2}}}\left[\frac{q_{1 \mathrm{to} 2} \mu_{1, k}}{\alpha_{1}}+\frac{q_{4 \mathrm{to} 2} \mu_{4, \mathrm{k}}}{\alpha_{4}}-\left(q_{2 \mathrm{to} 3}+q_{2 \mathrm{to} 8}+q_{2 \mathrm{to} 9}\right) \frac{\mu_{2, \mathrm{k}}}{\alpha_{2}}\right]+B-D \\
& \frac{\partial \mu_{3, k}}{\partial t}=\frac{1}{\overline{V_{3}}}\left[\frac{q_{2 \mathrm{to} 3} \mu_{2, k}}{\alpha_{2}}-\left(q_{3 \mathrm{to} 4}+q_{3 \mathrm{to} 5}\right) \frac{\mu_{3, k}}{\alpha_{3}}\right]+B-D \\
& \frac{\partial \mu_{4, k}}{\partial t}=\frac{1}{\overline{V_{4}}}\left[\frac{q_{3 \mathrm{to} 4} \mu_{3, \mathrm{k}}}{\alpha_{3}}+\frac{q_{6 \mathrm{to} 4} \mu_{6, k}}{\alpha_{6}}-\left(q_{4 \mathrm{to} 2}+q_{4 \mathrm{to} 7}\right) \frac{\mu_{4, k}}{\alpha_{4}}\right]+B-D \\
& \frac{\partial \mu_{5, k}}{\partial t}=\frac{1}{\overline{V_{5}}}\left[\frac{q_{3 \mathrm{to} 5} \mu_{3, k}}{\alpha_{3}}-\frac{q_{5 \mathrm{to} 6} \mu_{5, k}}{\alpha_{5}}\right]+B-D \\
& \frac{\partial \mu_{6, k}}{\partial t}=\frac{1}{\overline{V_{6}}}\left[\frac{q_{5 \text { to }} \mu_{5, k}}{\alpha_{5}}-\left(q_{6 \mathrm{to} 4}+q_{6 \mathrm{to} 7}\right) \frac{\mu_{6, k}}{\alpha_{6}}\right]+B-D \\
& \frac{\partial \mu_{7, k}}{\partial t}=\frac{1}{\overline{V_{7}}}\left[\frac{q_{6 \mathrm{to} 7} \mu_{6, k}}{\alpha_{6}}+\frac{q_{4 \mathrm{to} 7} \mu_{4, \mathrm{k}}}{\alpha_{4}}-\frac{q_{7 \mathrm{to} 1} \mu_{7, k}}{\alpha_{7}}\right]+B-D+S_{\text {sparger }, k} \\
& \frac{\partial \mu_{8, k}}{\partial t}=\frac{1}{\overline{V_{8}}}\left[\frac{q_{2 \mathrm{to} 8} \mu_{2, k}}{\alpha_{2}}-\left(q_{8 \mathrm{to9}}+q_{8 \mathrm{to} 10}\right) \frac{\mu_{8, k}}{\alpha_{8}}\right]+B-D \\
& \frac{\partial \mu_{9, k}}{\partial t}=\frac{1}{\overline{V_{9}}}\left[\frac{q_{2 \mathrm{to} 9} \mu_{2, k}}{\alpha_{2}}+\frac{q_{8 \mathrm{to} 9} \mu_{8, k}}{\alpha_{8}}-\left(q_{9 \mathrm{to} 12}+q_{9 \mathrm{to} 11}\right) \frac{\mu_{9, \mathrm{k}}}{\alpha_{9}}\right]+B-D \\
& \frac{\partial \mu_{10, k}}{\partial t}=\frac{1}{\overline{V_{10}}}\left[\frac{q_{8 \mathrm{to} 10} \mu_{8, k}}{\alpha_{8}}-\left(q_{10 \mathrm{to} 11}+q_{\mathrm{out} 10}\right) \frac{\mu_{10, k}}{\alpha_{10}}\right]+B-D \\
& \frac{\partial \mu_{11, k}}{\partial t}=\frac{1}{\overline{V_{11}}}\left[\frac{q_{9 \mathrm{to} 11} \mu_{9, k}}{\alpha_{9}}+\frac{q_{10 \mathrm{to} 11} \mu_{10, k}}{\alpha_{10}}-\left(q_{11 \mathrm{to} 12}+q_{\text {out } 11}\right) \frac{\mu_{11, k}}{\alpha_{11}}\right]+B-D \\
& \frac{\partial \mu_{12, k}}{\partial t}=\frac{1}{\overline{V_{12}}}\left[\frac{q_{11 \text { to } 12} \mu_{11, k}}{\alpha_{11}}+\frac{q_{9 \mathrm{to} 12} \mu_{9, k}}{\alpha_{9}}+\frac{q_{1 \mathrm{to} 12} \mu_{1, k}}{\alpha_{1}}-\left(q_{\text {out } 12}\right) \frac{\mu_{12, k}}{\alpha_{12}}\right]+B-D
\end{aligned}
$$

where $B$ and $D$ are the birth and death due to breakage and coalescence, similar to those in single compartment model in the supplementary data; see Eq. 7. The multi-compartment population balance was implemented using PD-QMOM in MATLAB; the ODE integrations were conducted with absolute and relative tolerances set at $10^{-8}$ for all solutions. The multi-compartment model represented by Eqs. 19 to 30 was solved using the ode113 solver in MATLAB. The simulations took about 5 minutes (wall clock) to complete on a GENIE workstation fitted with two dual-core 3.8 GHz Xeon processors and 3 GB RAM.

Figure 7 shows the evolution of bubble size for each compartment where the inter-compartment moment balances are calculated for this simulation. Unlike the single compartment where the steady-state is obtained within a second, the multi-compartment requires up 5 seconds at most to achieve steady-state bubble size. This is because the moment of evolution in neighbouring compartments can affect the evolution of the moments in another compartment resulting to a relatively longer time to approach steady-state. 

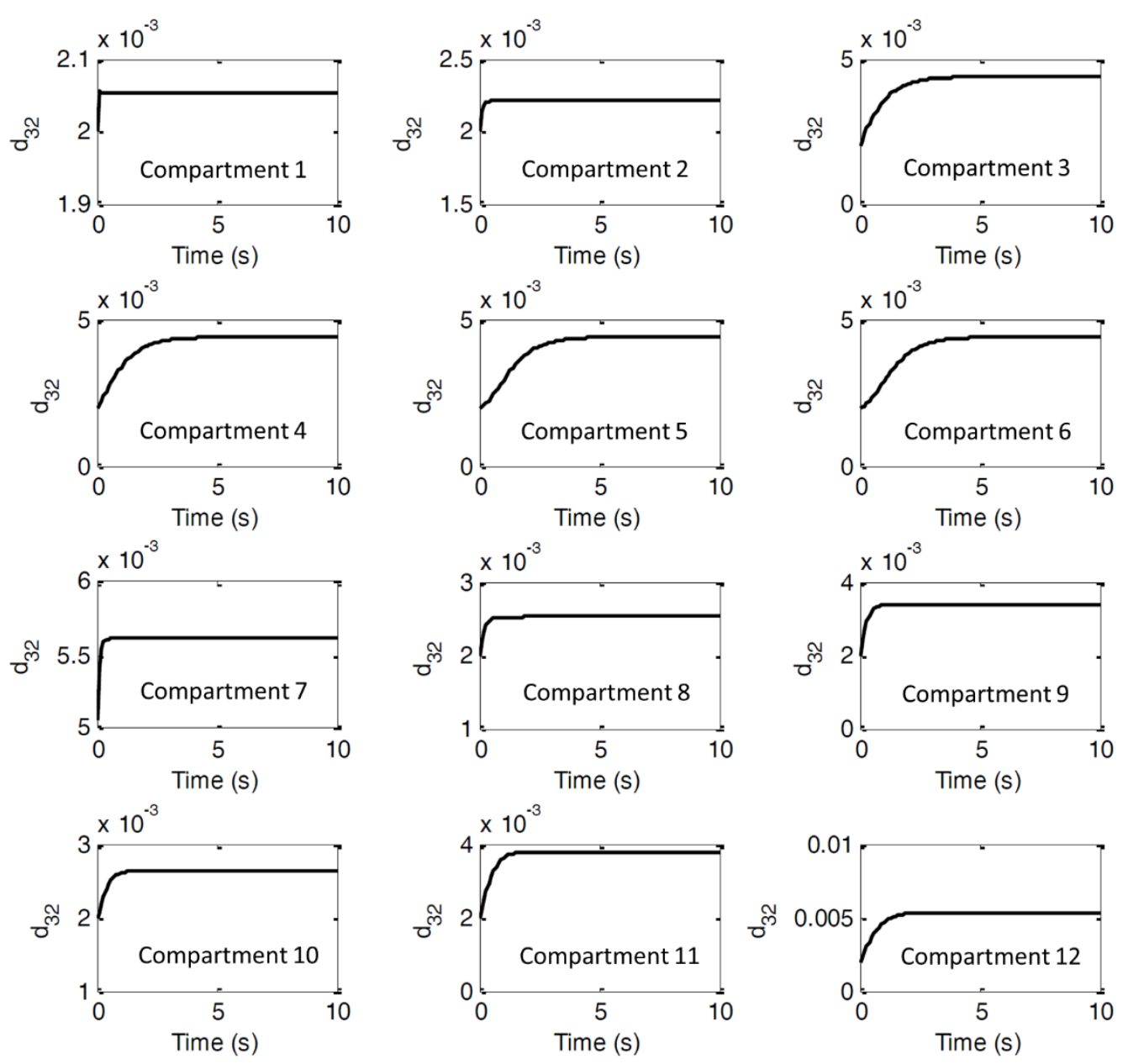

Figure 7: Evolution of the Sauter mean bubble size $d_{32}(\mathrm{~m})$ at each compartment.

Meanwhile, Figure 8 displays the prediction of the local steady-state bubble size via multicompartment PBM. The results show some qualitative agreement to Laakkonen et al. (2007)'s experiment data and Gimbun et al. (2009)'s CFD-PBM simulation. There are discrepancies on bubble size prediction in some compartments (e.g. compartment no. 3), where $d_{32}$ is slightly larger than the value measured by Laakkonen et al. (2007). Predictions of the multi-compartment model in this work were also in fair agreement with the CFD-PBM predictions from Gimbun et al. (2009). The discrepancy mainly occurs in the lower circulation loop where $d_{32}$ is overpredicted by the current model. This may be due to the small uniform bubble size (i.e. $2 \mathrm{~mm}$ ) employed throughout the tank for the initial CFD simulation which led to a higher gas hold-up around the lower circulation loop. It can be observed from Figure 8 that the assumptions of bubble size around $2 \mathrm{~mm}$ is only valid around the impeller and to some extent in the upper circulation loop but certainly not for lower circulation loop. It was concluded from the single compartment study that higher gas hold-up led to larger bubble size especially in regions of lower turbulence dissipation rate. Nevertheless the multi-compartment simulation has successfully reproduced the correct distribution of bubble size inside the tank with the smallest bubble harboring around the impeller region and the largest in the bulk flow of the upper circulation loop. The former is due high turbulence dissipation rate around the impeller region (refer to Table 1). This finding is in agreement with experimental measurements by Barigou and Greaves (1992) and Laakkonen et al. (2005) who also observed small bubble sizes around the impeller region. 


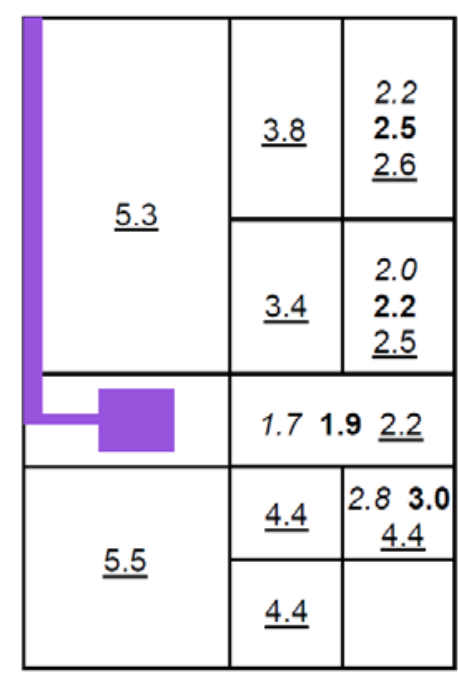

Figure 8: Prediction of local bubble size $\left(d_{32}\right)$. The italic font (CFD-PBM from Gimbun et al. (2009)), bold font (Laakkonen et al. (2007) experiment) and underlined font (this work).

There is also a growing concern regarding the use of constant bubble size assumption for gasliquid CFD simulation. Such an assumption is certainly not valid in a stirred tank where the turbulence dissipation rate gradient is high especially around the impeller region. The mean bubble size should be significantly smaller around the impeller region compared to the bulk region, as evidenced in the multi-compartment results. The bubble size can affect the prediction of turbulent flows, gas void fraction and the gas flow rate which is required for the multicompartment modelling. Thus it can be concluded that the error from the original CFD simulation can severely affect the results of the multi-compartment modelling. It is best that a four-way coupling is used to improve prediction accuracy in order to eliminate the assumption of uniform bubble size. It cannot be deny that the multi-compartment model is not capable of providing as high resolution as CFD, however they require less computational effort to simulate. Nevertheless, the multi-compartment model is capable of yielding a reasonably accurate prediction of the local bubble size, despite all its simplifications.

The gas hold-up is an important mass transfer parameter for gas-liquid stirred tanks. The gas hold-up in each compartment is related to the third moment and is estimated using Eq. S6. The gas hold-up obtained from the multi-compartment PBM is compared to the result from CFD simulation in Table 6. The prediction shows an excellent agreement between the multicompartment model and the CFD predictions, meaning that the third moment is perfectly conserved during the simulation thus confirming the validity of the multi-compartment model.

Table 6: Comparison between the gas hold-up from CFD simulation and the value obtained from multi-compartment simulation

\begin{tabular}{ccc}
\hline Compartment & $\begin{array}{c}\text { Multi-compartment } \\
\alpha_{g}=\pi \mu_{3} / 6 \times 100\end{array}$ & $\begin{array}{c}\text { CFD simulation } \\
\alpha_{g} \times 100\end{array}$ \\
\hline 1 & 7.56 & 7.56 \\
2 & 1.16 & 1.16 \\
3 & 1.56 & 1.56 \\
4 & 0.07 & 0.07 \\
5 & 0.02 & 0.02 \\
6 & 0.00 & 0.00 \\
\hline
\end{tabular}




\begin{tabular}{ccc}
\hline 7 & 1.53 & 1.53 \\
8 & 1.44 & 1.44 \\
9 & 3.92 & 3.92 \\
10 & 0.47 & 0.47 \\
11 & 2.14 & 2.14 \\
12 & 3.72 & 3.72 \\
\hline
\end{tabular}

\section{Conclusion}

The PBM implementation in a single compartment model (refer to supplementary data) has demonstrated the capability of the PD-QMOM algorithm, with realistic breakage and coalescence kernels in predicting the evolution of bubble size in a homogeneous gas-liquid flow. The prediction from the single compartment PBM shows a reasonable agreement with the Sauter mean bubble sizes obtained from empirical correlations. The algorithm also responded well to changes in the turbulence dissipation rate and the initial BSD. The results suggest that the final bubble size is only affected by the turbulence dissipation rate and local gas hold-up, but is not affected by the initial bubble size. The single compartment model was combined with gas-liquid CFD simulation to form a multi-compartment model. The multi-compartment PBM yielded a reasonable prediction of the local bubble size and compared with experimental measurements by Laakkonen et al. (2007). The three-way coupling PBM requires less computational effort and easier to converge than that of four-way coupling. Thus, the model developed and tested in this work may be useful for a quick evaluation of local bubble size in an aerated stirred tank.

\section{References}

Alopaeus V, Koskinen J, Keskinen KI. Simulation of the population balances for liquid-liquid systems in a nonideal stirred tank. Part 1: Description and qualitative validation of the model. Chem. Eng. Sci. 1999;54(24):5887-5899.

Alves SS, Maia CI, Vasconcelos JMT. Experimental and modelling study of gas dispersion in a double turbine stirred tank. Chem. Eng. Sci. 2002;57(3):487-496.

Andersson R, Andersson B. On the breakup of fluid particles in turbulent flows. AIChE J. 2006;52(6):2020-2030.

Bakker A, Van den Akker H. A computational model for the gas-liquid flow in stirred reactors. Che. Eng. Res. Des. 1994;72(A4):594-606.

Barigou M, Greaves M. Bubble-size distributions in a mechanically agitated gas-liquid contactor. Chem. Eng. Sci. 1992;47(8):2009-2025.

Behzadi A, Issa RI, Rusche H. Modelling of dispersed bubble and droplet flow at high phase fractions. Chem. Eng. Sci. 2004;59(4):759-770.

Buffo A, Vanni M, Marchisio DL. Multidimensional population balance model for the simulation of turbulent gas-liquid systems in stirred tank reactors. Chem. Eng. Sci. 2012;70:31-44.

Bombač, A, Žun, I, Filipič, B, Žumer, M. (1997). Gas-filled cavity structures and local void fraction distribution in aerated stirred vessel. AIChE J. 1997;43(11):2922-2931.

Clift R, Grace JR, Weber ME. Bubbles, drops, and particles. New York: Academic Press; 1978.

Deen NG. An experimental and computational study of fluid dynamics in gas-liquid chemical reactors (Doctoral dissertation). Esbjerg: Aalborg University;2001

Deen NG, Solberg T, Hjertager BH. Flow generated by an aerated rushton impeller: two-phase PIV experiments and numerical simulations. Can. J. Chem. Eng. 2002;80(4):1-15.

Degaleesan S. Fluid dynamic measurements and modeling of liquid mixing in bubble columns (Doctoral dissertation). St. Louis MO: Washington University; 1997.

Fluent Inc. User’s Guide. Lebanon, New Hampshire; 2005.

Gimbun J. Scale-up of gas-liquid stirred tanks using coupled computational fluid dynamics and population balance modelling (Doctoral dissertation). Leicestershire: Loughborough University;2009. 
Gimbun J, Rielly CD, Nagy ZK. Modelling of mass transfer in gas-liquid stirred tanks agitated by Rushton turbine and CD-6 impeller: A scale-up study. Chem. Eng. Res. Des. 2009;87(4):437-451.

Gordon RG. Error bounds in equilibrium statistical mechanics. J. Math Phys.1968;9:655-663.

Hristov H, Mann R, Lossev V, Vlaev SD, Seichter P. A 3-D analysis of gas-liquid mixing, mass transfer and bioreaction in a stirred bio-reactor. Food Bioprod. Process. 2001;79(4):232241.

Ishii M, Zuber N. Drag coefficient and relative velocity in bubbly, droplet or particulate flows. AIChE J. 1979;25(5):843-855.

Kennard EH. Theory of gasses. New York: Mc Graw-Hill; 1938.

Kerdouss F, Bannari A, Proulx P. CFD modeling of gas dispersion and bubble size in a double turbine stirred tank. Chem. Eng. Sci. 2006;61(10):3313-3322.

Khan, FR, Rielly, CD, Brown, DAR. Angle-resolved stereo-PIV measurements close to a downpumping pitched-blade turbine. Chem. Eng. Sci. 2006;61(9):2799-2806.

Khopkar AR, Ranade VV. CFD simulation of gas-liquid stirred vessel: VC, S33, and L33 flow regimes. AIChE J. 2006;52(5):1654-1672.

Kolmogorov AN. The local structure of turbulence in incompressible viscous fluid for very large Reynolds numbers. Dokl. Akad. Nauk SSSR. 1941;30(4):301-305.

Kresta SM, Etchells III AW, Dickey DS, Atiemo-Obeng VA. Advances in industrial mixing: a companion to the handbook of industrial mixing. New Jersey: John Wiley \& Sons Inc; 2015. ISBN: 978-0-470-52382-7.

Laakkonen M, Moilanen P, Miettinen T, Saari K, Honkanen M, Saarenrinne P, Aittamaa J. Local bubble size distributions in agitated vessel comparison of three experimental techniques. Chem. Eng. Res. Des. 2005;83(1):50-58.

Laakkonen M, Moilanen P, Alopaeus V, Aittamaa J. Dynamic modeling of local reaction conditions in an agitated aerobic fermenter. AIChE J. 2006;52(5):1673-1689.

Laakkonen M, Alopaeus V, Aittamaa J. Validation of bubble breakage, coalescence and mass transfer models for gas-liquid dispersion in agitated vessel. Chem. Eng. Sci. 2006;61(1):218-228.

Laakkonen M, Moilanen P, Alopaeus V, Aittamaa J. Modelling local bubble size distributions in agitated vessels. Chem. Eng. Sci. 2007;62(3):721-740.

Lane GL, Schwarz MP, Evans GM. Predicting gas-liquid flow in a mechanically stirred tank. Appl. Math. Model. 2002;26(2):223-235.

Lane GL, Schwarz MP, Evans GM. Numerical modelling of gas-liquid flow in stirred tanks. Chem. Eng. Sci. 2005;60(8-9):2203-2214

Marchisio DL, Vigil RD, Fox RO. Quadrature method of moments for aggregation-breakage processes. J. Colloid Interface Sci. 2003;258(2):322-334.

Montante G, Horn D, Paglianti A. Gas-liquid flow and bubble size distribution in stirred tanks. Chem. Eng. Sci. 2008;63(8):2107-2118.

Morud KE, Hjertager BH. LDA measurements and CFD modelling of gas-liquid flow in a stirred vessel. Chem. Eng. Sci. 1996;51(2):233-249.

Petitti M, Vanni M, Marchisio DL, Buffo A, Podenzani F. Simulation of coalescence, break-up and mass transfer in a gas-liquid stirred tank with CQMOM. Chem. Eng. J. 2013;228:11821194.

Pohorecki R, Moniuk W, Bielski P, Sobieszuk P. Diameter of bubbles in bubble column reactors operating with organic liquids. Chem. Eng. Res. Des. 2005;83(7):827-832.

Prince MJ, Blanch HW. Bubble coalescence and break-up in air-sparged bubble columns. AIChE J. 1990;36(10):1485-1499.

Rotta JC. Turbulent flows (Turbulente Stromungen). Stuttgart: Teubner-Verlag; 1972.

Scargiali F, D’Orazio A, Grisafi F, Brucato A. Modelling and simulation of gas-liquid hydrodynamics in mechanically stirred tanks. Chem. Eng. Res. Des. 2007;85(5):637-646. 
Schiller L, Naumann L. A drag coefficient correlation. Z. Ver. Deutsch. Ing. 1935;77:318. Shimizu K, Takada S, Minekawa K, Kawase Y. Phenomenological model for bubble column reactors: prediction of gas hold-ups and volumetric mass transfer coefficients. Chem. Eng. J. 2000;78(1):21-28.

Smith, JM. Large multiphase reactors: some open questions. Chem. Eng. Res. Des. 2006;84(4):265-271.

Sun H, Mao Z, Yu G. Experimental and numerical study of gas hold-up in surface aerated stirred tanks. Chem. Eng. Sci. 2006;61(12):4098-4110.

Venneker BCH, Derksen JJ, Van den Akker H. Population balance modeling of aerated stirred vessels based on CFD. AIChE J. 2002;48(4):673-685.

Wang W, Mao Z, Yang C. Experimental and numerical investigation on gas holdup and flooding in an aerated stirred tank with Rushton impeller. Ind. Eng. Chem. Res. 2006;45(3):11411151.

Wilkinson PM. Physical aspects and scale-up of high pressure bubble columns (Doctoral dissertation). The Netherlands: University of Groningen; 1991.

Wu, H, Patterson, G.K. Laser-doppler measurements of turbulent-flow parameters in a stirred mixer. Chem. Eng. Sci. 1989;44(10):2207-2221.

Zahradník J, Mann R, Fialová M, Vlaev D, Vlaev SD, Lossev V, Seichter P. A networks-of-zones analysis of mixing and mass transfer in three industrial bioreactors. Chem. Eng. Sci. 2001;56(2):485-492.

Zhou, G., \& Kresta, S. M. (1996). Distribution of energy between convective and turbulent-flow for 3 frequently used impellers. Chem. Eng. Res. Des. 1996;74(3):379-389. 